\title{
Exponential Fitting of Matricial Multistep Methods for Ordinary Differential Equations
}

\author{
By E. F. Sarkany and W. Liniger
}

\begin{abstract}
We study a class of explicit or implicit multistep integration formulas for solving $N \times N$ systems of ordinary differential equations. The coefficients of these formulas are diagonal matrices of order $N$, depending on a diagonal matrix of parameters $Q$ of the same order. By definition, the formulas considered here are exact with respect to $y^{\prime}=-D y+\phi(x, y)$ provided $Q=h D, h$ is the integration step, and $\phi$ belongs to a certain class of polynomials in the independent variable $x$. For arbitrary step number $k \geqslant 1$, the coefficients of the formulas are given explicitly as functions of $Q$. The present formulas are generalizations of the Adams methods $(Q=0)$ and of the backward differentiation formulas $(Q=+\infty)$. For arbitrary $Q$ they are fitted exponentially at $Q$ in a matricial sense. The implicit formulas are unconditionally fixed- $h$ stable. We give two different algorithmic implementations of the methods in question. The first is based on implicit formulas alone and utilizes the Newton-Raphson method; it is well suited for stiff problems. The second implementation is a predictor-corrector approach. An error analysis is carried out for arbitrarily large $Q$. Finally, results of numerical test calculations are presented.
\end{abstract}

1. Introduction. This paper is concerned with a class of integration formulas for $N \times N$ systems of ordinary differential equations proposed earlier by one of the authors [1]. These formulas are of linear multistep type,

$$
\sum_{j=-(k-1)}^{1} \alpha_{j+k-1}(Q) y_{n+j}-h \sum_{j=-(k-1)}^{1} \beta_{j+k-1}(Q) y_{n+j}^{\prime}=0 .
$$

Their coefficients $\alpha_{j}(Q)$ and $\beta_{j}(Q)$ are diagonal matrices of order $N$ depending on a diagonal matrix $Q$ of parameters of the same order. By the symbol $\mathbf{F}_{k}$ we refer to the explicit ("predictor") $k$-step formula $\left(\beta_{k}=0\right)$ and by $\mathbf{F}_{k}^{*}$ to the implicit ("corrector") formula $\left(\beta_{k} \neq 0\right)$ of the same length. Similarly, $F_{k}$ and $F_{k}^{*}$ denote generic scalar components (rows) of $\mathbf{F}_{k}$ and $\mathbf{F}_{k}^{*}$, respectively. By definition, $\mathbf{F}_{k}$ or $\mathbf{F}_{k}^{*}$ are exact, for arbitrary fixed $Q$, when applied to

$$
y^{\prime}=-D y+\phi(x, y),
$$

Received March 12, 1973, revised January 8, 1974.

AMS (MOS) subject classifications (1970). Primary 65L05, 65D30; Secondary 39A35.

Key words and phrases. Ordinary differential equations, matricial multistep methods, exponential fitting, unconditional fixed-h stability.

Copyright $\odot 1974$, American Mathematical Society 
with $Q=h D$, provided $\phi(x, y(x))$ is any polynomial in the independent variable $x$ of degree $\leqslant k-1$ or $\leqslant k$, respectively. Each row $F_{k}$ or $F_{k}^{*}$ depends solely on $q=h d$, where $d$ and $q$ are the corresponding diagonal entries of $D$ and $Q$, respectively. Systems of the form (1.2) arise naturally in applications, e.g., in nuclear reactor calculations [2]. The approach taken in this paper is related to those of Cohen and Flatt [3] and of Guderley and Hsu [4].

Any $N \times N$ system

$$
y^{\prime}=f(x, y)
$$

takes, of course, the form (1.2) if we let $\phi=D y+f$. However, as one would expect, the formulas considered here lend themselves particularly well to problems in which $\phi$ is in some sense small compared to $f$ or to $D y$. Even so the present formulas remain meaningful and competitive with well-known conventional methods when parametrized by $\widetilde{Q}=h \widetilde{D}, \widetilde{D} \neq D$, rather than by $Q$. In fact, for $q=0, F_{k}$ and $F_{k}^{*}$ are simply the explicit and implicit $k$-step Adams formulas, respectively. Similarly, subject to appropriate scaling, $F_{k}^{*}$ for $q=+\infty$ is the $k$-step backward differentiation formula (BDF). For arbitrary $q, 0 \leqslant q \leqslant+\infty, F_{k}^{*}$ is exponentially fitted [5] at $q=h d$. Nevertheless, a certain amount of caution is needed in fitting at locations $\widetilde{Q} \neq Q$, as explained in the second-to-last paragraph of Section 3.

The formulas $\mathbf{F}_{k}^{*}$ are useful for integrating stiff [6] systems because, when fitted at large $q$ 's, the $F_{k}^{*}$ possess some of the strong fixed- $h$ stability properties required to control the corresponding "rapid transients". More specifically, $F_{1}^{*}$ is $A$ stable [7] for any $q, 0 \leqslant q \leqslant+\infty . F_{2}^{*}$ is $A$-stable for any $q, 2<q \leqslant+\infty$. $F_{3}^{*}$ is $A(\alpha)$-stable [8] and stiffly stable [9] for any $q \geqslant 5$. For arbitrary $k, F_{k}^{*}$ is $A_{\infty}$-stable [10] for any sufficiently large $q$. On the other hand, when fitted at sufficiently small values of $|q|, F_{k}$ and $F_{k}^{*}$ are stable [11] for arbitrary $k$ and thus well suited for treating nonstiff components. More specifically, $F_{3}^{*}$ is stable for all $q, 0 \leqslant q \leqslant+\infty$.

If the implicit formula $\mathbf{F}_{k}^{*}$ is applied to (1.2) or (1.3), a set of algebraic equations, in general nonlinear, must be solved at every time step. It has been found [12] that, in dealing with stiff problems, the Newton-Raphson (NR) method is useful for accomplishing this task. The iteration can be started, e.g., from a first guess obtained by polynomial extrapolation from previous solution values. ${ }^{*}$ With a sufficiently accurate first guess, only one or two NR steps need to be carried out to make the stopping error of the iteration compatible with the local truncation error [13]. The fixed-h

*Obviously, with large integration steps, this technique is meaningful primarily during the asymptotic phase of the solution; i.e., after the stiff components have been damped out. During the initial, transient phase, it is appropriate to use small integration steps to sample the rapidly varying solutions. 
stability analysis of the formulas $F_{k}^{*}, k \geqslant 1$, given in this paper remains valid even when we use one single NR iteration step. The reason for it is that this analysis is carried out relative to a linear test equation [7],

$$
y^{\prime}=\lambda y, \quad \lambda=\text { const },
$$

and that for linear equations the NR method converges in one step.

Some iterative methods of solving the difference equations other than the NR technique are sensitive to stiffness and in its presence become inefficient. This is the case, e.g., for the straight Picard iteration [12]. Similarly, as one can deduce from the results of [4], it is not recommendable to use finite predictor-corrector (PC) algorithms in solving stiff problems because, with such algorithms, the unconditional fixed- $h$ stability properties of the $F_{k}^{*}$ are lost [12]. This is, of course, due to the explicit character of such finite PC methods [7]. However, as mentioned in the last section of this paper, exponentially fitted, finite PC methods are useful for solving nonstiff problems with relatively large integration steps.

A class of parametrized multistep methods similar to the one considered here is described in [14]. Those methods have step number $k$ and order of accuracy $p=2 k$, and are said to be $A$-stable. For $k \geqslant 1$ this contradicts the well-known constraint $p \leqslant 2$ for $A$-stability [7]. The discrepancy stems from the fact that in [14], as well as in [15], the term $A$-stability is, somewhat misleadingly, used to identify a much weaker stability property than in [7]. In its original spirit, this term was used relative to an integration formula with fixed coefficients, applied to the test equation (1.4) with arbitrary complex $\lambda, \operatorname{Re} \lambda<0$. Similarly, in the present paper, fixed- $h$ stability analysis is carried out for given fixed values of $d$ or $q$, and thus fixed coefficients, but for an arbitrary complex $\lambda$ which varies independently of $d$. As opposed to this, the term $A$-stability in [14] and [15] means fixed- $h$ stability for any $\lambda, \operatorname{Re} \lambda<0$, of a parametrized formula whose coefficients, for each $\lambda$, are exponentially fitted at $\lambda h$. In other words, the results of [14] and [15] are restricted to the special case $d=\lambda$.

The outline of the present paper is as follows: In Section 2 we give explicit expressions for the coefficients of $\mathbf{F}_{k}$ and $\mathbf{F}_{k}^{*}$ for arbitrary $k$. We discuss the relationship between these formulas and Adams methods, BDF, and exponentially fitted formulas in the sense of [5]. In Section 3 we study unconditional fixed-h stability and stability of the formulas $F_{k}^{*}$. Section 4 is devoted to algorithmic aspects. We define a one-step Newton-Raphson implementation of $\mathbf{F}_{\mathbf{3}}^{*}$. Then we analyze the local truncation error of the formulas $\mathbf{F}_{k}$ and $\mathbf{F}_{k}^{*}$. The result of this analysis is used to define $\mathrm{PC}$ algorithms. Finally, in Section 5, we describe numerical results obtained for various test problems. The performance of the methods considered here compares favorably with that of other existing methods.

2. Derivation of Integration Formulas. A. The analysis of parts A and B of this section is presented in more detail in [16]; it is similar to the one given in [17]. 
Let $\left\{x_{n}\right\}, x_{n}=n h, n=0,1, \cdots, h>0$. Equation (1.2) can be converted into an integral equation,

$$
y\left(x_{n+1}\right)=e^{-h D} y\left(x_{n}\right)+\int_{x_{n}}^{x_{n+1}} e^{-\left(x_{n+1}-x\right) D} \phi(x, y(x)) d x,
$$

where $x_{n}$ is fixed, $x_{n+1}$ represents the independent variable, and $h=x_{n+1}-x_{n}$. With (2.1) we can associate a class of linear multistep formulas. To do this we approximate $\phi(x, y)$ by a polynomial in $x$ and evaluate the integral in closed form. If the polynomial is defined by Lagrange interpolation through $\phi_{n-i}=\phi\left(x_{n-i}, y_{n-i}\right), i=0$, $1, \cdots, k-1, k \geqslant 1$, this procedure results in a $k$-step formula $\mathbf{F}_{k}$ of explicit or predictor type. If, on the other hand, Lagrange interpolation through $\phi_{n-i+1}, i=0$, $\cdots, k$, is used, an implicit or corrector formula $\mathbf{F}_{k}^{*}$ with the same number of steps is obtained.

In order to derive $F_{k}$, we use Newton's backward difference formula [18] to fit a polynomial $\hat{\phi}(x)$ through $\phi_{n-i}, i=0, \cdots, k-1$. If in (2.1) we replace $\phi$ by $\hat{\phi}$ we obtain the difference form of $F_{k}$,

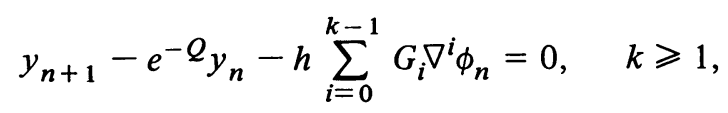

where $\nabla^{i}$ denotes the $i$ th backward difference, $Q=h D$, and

$$
G_{i}=(-1)^{i} \int_{0}^{1} e^{-(1-\xi) Q}\left(\begin{array}{c}
-\xi \\
i
\end{array}\right) d \xi, \quad i=0,1, \cdots .
$$

Upon evaluation of the integral [19] this yields

$$
G_{i}=\sum_{j=0}^{i} \psi_{i j} Q^{-(j+1)}-e^{-Q} \sum_{j=0}^{i} \xi_{i j} Q^{-(j+1)}, \quad i \geqslant 0,
$$

where

$$
\begin{aligned}
\psi_{i j} & =\frac{j !}{i !} \gamma_{i j}, \quad i \geqslant 0,0 \leqslant j \leqslant i, \\
\xi_{i j} & =\frac{1}{i !} \sum_{l=j}^{i} \gamma_{i l} l ! /(l-j) !, \quad 0 \leqslant j \leqslant i,
\end{aligned}
$$

and where the $\gamma_{i j}, i \geqslant 0,0 \leqslant j \leqslant i$, are defined by

$$
\left(\begin{array}{c}
-\xi \\
i
\end{array}\right) \equiv \frac{(-1)^{i}}{i !} \sum_{j=0}^{i} \gamma_{i j} \xi^{j}, \quad i \geqslant 0,
$$

with $\zeta=1-\xi$. Obviously, $\gamma_{0,0}=\gamma_{1,0}=1$ and $\gamma_{1,1}=-1$. It is easy to show that the $\gamma_{i j}$ satisfy the recurrence relations

$$
\left.\begin{array}{rl}
\gamma_{i+1,0} & =(i+1) \gamma_{i, 0}, \\
\gamma_{i+1, i+1} & =-\gamma_{i i}, \\
\gamma_{i+1, j} & =(i+1) \gamma_{i j}-\gamma_{i, j-1}, \quad 1 \leqslant j \leqslant i
\end{array}\right\}, \quad i \geqslant 1 .
$$


Note that

$$
\sum_{i=0}^{i} \gamma_{i j}=0, \quad i \geqslant 1,
$$

which follows from (2.7) for $\zeta=1$. From (2.9) it follows that $\xi_{i, 0}=0, i>1$.

To transform $\mathbf{F}_{k}$ into ordinate form, we make the substitution

$$
\nabla^{i} \phi_{n}=\sum_{j=0}^{i}(-1)^{i}\left(\begin{array}{l}
i \\
j
\end{array}\right) \phi_{n-j}, \quad i=0,1, \cdots \text {. }
$$

This yields

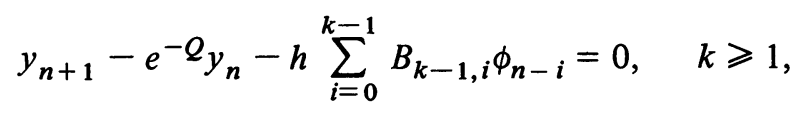

where

$$
\begin{aligned}
B_{i j} & =(-1)^{j} \sum_{l=j}^{i}\left(\begin{array}{l}
l \\
j
\end{array}\right) G_{l} \\
& =\sum_{l=0}^{i} \mu_{i j l} Q^{-(l+1)}-e^{-Q} \sum_{l=0}^{i} \nu_{i j l} Q^{-(l+1)}, \quad i \geqslant 0,0 \leqslant j \leqslant i,
\end{aligned}
$$

and where

$$
\left\{\begin{array}{c}
\mu \\
\nu
\end{array}\right\}_{i j l}=(-1)^{j} \sum_{r=\max (j, l)}^{i}\left(\begin{array}{l}
r \\
j
\end{array}\right)\left\{\begin{array}{l}
\psi \\
\xi
\end{array}\right\}_{r l,} \quad 0 \leqslant j \leqslant i, \quad 0 \leqslant l \leqslant i,
$$

meaning that $\mu$ and $\nu$ are associated with $\psi$ and $\xi$, respectively.

B. An analysis very similar to the one given above for the formulas $\mathbf{F}_{\boldsymbol{k}}$ can be carried out for the $\mathbf{F}_{k}^{*}$. All quantities associated with these implicit formulas are so identified by asterisks. The difference form of $\mathbf{F}_{k}^{*}$ is

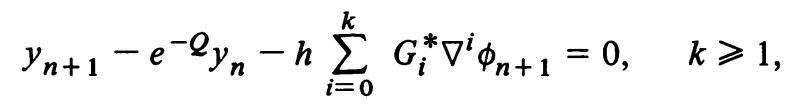

where

$$
G_{i}^{*}=(-1)^{i} \int_{-1}^{0} e^{\xi Q}\left(\begin{array}{c}
-\xi \\
i
\end{array}\right) d \xi
$$

$$
=\sum_{j=0}^{i} \psi_{i j}^{*} Q^{-(j+1)}-e^{-Q} \sum_{j=0}^{i} \xi_{i j}^{*} Q^{-(j+1)}, \quad i=0,1, \cdots
$$

Here

$$
\left.\begin{array}{l}
\psi_{i j}^{*}=(-1)^{j} \frac{j !}{i !} \gamma_{i j}^{*} \\
\xi_{i j}^{*}=\frac{1}{i !} \sum_{l=j}^{i}(-1)^{l} \gamma_{i l}^{*} \frac{l !}{(l-j) !}
\end{array}\right\}, \quad 0 \leqslant j \leqslant i
$$

and it is easy to show that the quantities $\gamma_{i j}^{*}$, which are defined by the relation 


$$
\left(\begin{array}{c}
-\xi \\
i
\end{array}\right) \equiv \frac{(-1)^{i}}{i !} \sum_{j=0}^{i} \gamma_{i j}^{*} \xi^{j}, \quad i \geqslant 0
$$

satisfy $\gamma_{i j}^{*}=\xi_{i j}$ for all $i, j$. By definition, $\xi_{i, 0}^{*}=0$ for $i \geqslant 2$.

The relation (2.13) is equivalent to

$$
y_{n+1}-e^{-Q} y_{n}-h \sum_{i=0}^{k} B_{k i}^{*} \phi_{n+1-i}=0, \quad k \geqslant 1,
$$

the ordinate form of $\mathbf{F}_{k}^{*}$, where

$$
B_{i j}^{*}=(-1)^{j} \sum_{l=j}^{i}\left(\begin{array}{l}
l \\
j
\end{array}\right) G_{l}^{*}, \quad i \geqslant 0,0 \leqslant j \leqslant i .
$$

The relationship (2.18) between the $B_{i j}^{*}$ and $G_{l}^{*}$ is the same as that between the $B_{i j}$ and $G_{l}$, respectively. The equations $(2.11)$ and $(2.12)$ thus remain valid between the "starred" quantities.

In the limit where a component $q$ of $Q$ tends to zero, the calculation by the closed expressions given in this section, of the components $g_{i}(q)$ and $g_{i}^{*}(q)$ of $G_{i}(Q)$ and $G_{i}^{*}(Q)$, respectively, is affected by roundoff errors. In this limit it is preferable to compute the quantities $g_{i}$ and $g_{i}^{*}$ by power series expansions as mentioned in Section 4 below.

C. The formulas considered in this paper are generalizations of conventional Adams formulas. More specifically, in the limit $q \rightarrow 0, F_{k}$ or $F_{k}^{*}$ tend to the explicit or implicit $k$-step scalar Adams formula, respectively. In fact, it is shown in Section 4 that, if $q$ is treated as an independent, constant parameter, the local truncation error of $F_{k}^{*}$ is $q g_{k+1}^{*} h^{k+1} y_{n}^{(k+1)}+O\left(h^{k+2}\right)$. Therefore, for $q=0$, the order of accuracy [20] of $F_{k}^{*}$ is $p=k+1$. Also, for $q=0$, we have $\phi=y^{\prime}$ according to (1.2) and thus $F_{k}^{*}$ is of Adams type; i. e., it involves the two leading terms only of $y$. Because of uniqueness [21], this formula is, in fact, the well-known $k$-step implicit Adams formula. A similar argument shows that, for $q=0$, the formula $F_{k}$ is the explicit $k$-step Adams formula of order $p=k$.

Now consider the limit $q \rightarrow+\infty$. Here, if we neglect exponentially small terms versus negative powers of $q$, we find that $g_{0}^{*}=\psi_{0,0}^{*} q^{-1}=q^{-1}$ and $g_{i}^{*}=\psi_{i, 1}^{*} q^{-2}+$ $O\left(q^{-3}\right), i \geqslant 1$. Therefore, if we let

$$
\begin{aligned}
\theta_{k, 0}^{*} & =\sum_{i=1}^{k} \psi_{l, 1}^{*}, \\
\theta_{k j}^{*} & =(-1)^{j} \sum_{l=j}^{k}\left(\begin{array}{l}
l \\
j
\end{array}\right) \psi_{l, 1}^{*}, \quad 1 \leqslant j \leqslant k,
\end{aligned}
$$

and if by $b_{k j}^{*}$ we denote a generic diagonal component of $B_{k j}^{*}$, we have

$$
\begin{aligned}
b_{k, 0}^{*} & =q^{-1}+\theta_{k, 0}^{*} q^{-2}+O\left(q^{-3}\right), \\
b_{k j}^{*} & =\theta_{k j}^{*} q^{-2}+O\left(q^{-3}\right), \quad 1 \leqslant j \leqslant k .
\end{aligned}
$$


Thus, since $h \phi_{n+1-j}=h y_{n+1-j}^{\prime}+q y_{n+1-j}$, one finds

$$
\begin{aligned}
b_{k, 0}^{*} h \phi_{n+1} & =y_{n+1}+\theta_{k, 0}^{*} q^{-1} y_{n+1}+h q^{-1} y_{n+1}^{\prime}+O\left(q^{-2}\right), \\
b_{k j}^{*} h \phi_{n+1-j} & =\theta_{k j}^{*} q^{-1} y_{n+1-j}+O\left(q^{-2}\right), \quad 1 \leqslant j \leqslant k,
\end{aligned}
$$

and, after multiplying the formula $F_{k}^{*}$ through by $q$, this formula, in the limit $q \rightarrow+\infty$, becomes

$$
\sum_{j=0}^{k} \alpha_{k-j} y_{n+1-j}-h y_{n+1}^{\prime}=0
$$

where the coefficients $\alpha_{i}, i=0, \cdots, k$, depend of course on $k$. Formula (2.19) is of BDF type; i.e., it contains only one single $y^{\prime}$-term, the leading one. But for any $q \neq 0$ the truncation error of $F_{k}^{*}$ given above is $O\left(h^{k+1}\right)$, i. e., $F_{k}^{*}$ has order $p=k$. Again by uniqueness [21], the formula (2.19) is thus identical with the standard $k$-step BDF, normalized to $\beta_{k}=1$.

Finally, by a similar argument as in the case $q \rightarrow+\infty$, one concludes that for any $q, 0<q<+\infty$, the component formula $F_{k}^{*}$ is. identical with the general $k$-step formula fitted exponentially at $q$ in the sense of [5].

3. Stability Analysis. In proving $A$-stability [7] for linear multistep formulas we may, according to [22], test the two sufficient conditions $\mathrm{N} 1:\left|\sigma_{i}\right|<1, i=1$, $\cdots, k$, where $\sigma_{i}$ are the roots of $\sigma(w)$, and $\mathrm{N} 2: \operatorname{Re}[\rho(w) / \sigma(w)] \geqslant 0$ for all $w$, $|w|=1$. Via the transformations $w=w(z)=(z+1) /(z-1), r(z)=(z-1)^{k} \rho(w(z))$, and $s(z)=(z-1)^{k} \sigma(w(z))$, condition $\mathrm{N} 1$ takes the form $N 1^{\prime}: \operatorname{Re} s_{i}<0, i=1$, $\cdots, k$, where the $s_{i}$ are the roots of $s(z)$. N1' can be tested by the Routh-Hurwitz theory [23]. One writes $s(z)=a_{0} z^{k}+b_{0} z^{k-1}+a_{1} z^{k-2}+\cdots$ and denotes by $a_{0}, b_{0}, c_{0}, \cdots$ the entries of the first column of the Routh tableau. Then $\mathrm{N} 1^{\prime}$ is satisfied if and only if $a_{0}, b_{0}, c_{0}, \cdots$ are all nonzero and of equal sign. In discussing fixed- $h$ stability with respect to the test equation (1.4) we use the notation $q^{\prime}=$ $\lambda h$, as distinguished from the fitting location, $q=d h$ (see second-to-last paragraph of Introduction).

The formula

$$
\begin{aligned}
F_{1}^{*}: & -y_{n}+y_{n+1} \\
& -h\left\{\left[q^{-1}-\left(e^{q}-1\right)^{-1}\right] y_{n}^{\prime}+\left[\left(1-q^{-1}\right)+\left(e^{q}-1\right)^{-1}\right] y_{n+1}^{\prime}\right\}=0
\end{aligned}
$$

is $A$-stable for any $q, 0 \leqslant q \leqslant+\infty$. In fact, as is shown in [12], the exponentially fitted, weighted Euler formula possesses this property and, according to the previous section, the latter and $F_{1}^{*}$ are equivalent.

In [5] it is stated without proof that, for $2<q \leqslant+\infty$, the exponentially fitted two-step formula of order $p=2$, which is equivalent to 


$$
\begin{aligned}
F_{2}^{*}:[(1- & \left.\left.2 q^{-1}\right)+e^{-q}\left(1+2 q^{-1}\right)\right] y_{n-1}+4\left[\left(-1+q^{-1}\right)-e^{-q} q^{-1}\right] y_{n} \\
+ & {\left[\left(3-2 q^{-1}\right)+e^{-q}\left(-1+2 q^{-1}\right)\right] y_{n+1} } \\
- & \left\{\left[\left(-q^{-1}+2 q^{-2}\right)-e^{-q}\left(q^{-1}+2 q^{-2}\right)\right] y_{n-1}^{\prime}\right. \\
+ & {\left[\left(4 q^{-1}-4 q^{-2}\right)-e^{-q}\left(2-4 q^{-2}\right)\right] y_{n}^{\prime} } \\
+ & {\left.\left[\left(2-3 q^{-1}+2 q^{-2}\right)-e^{-q}\left(-q^{-1}+2 q^{-2}\right)\right] y_{n+1}^{\prime}\right\}=0 }
\end{aligned}
$$

is $A$-stable. Here we give a proof: We find ${ }^{* *} a_{0}=g_{0}^{*}=\left[\left(1-e^{-q}\right) / q\right]>0, q>0$. Let $\hat{b}_{0}=q^{2} b_{0} / 2=q-\left(1-e^{-q}\right)$, where $b_{0}=2\left(g_{0}^{*}+g_{1}^{*}\right)$. Then $\hat{b}_{\dot{0}}^{\prime}(q)>0$, $q>0$, and $\hat{b}_{0}(0)=0$. Thus $\hat{b}_{0}(q)>0, q>0 \Rightarrow b_{0}(q)>0, q>0$. Finally, let $\hat{c}_{0}=q^{3} c_{0}=(2-q)\left[(2-q)-(2+q) e^{-q}\right]$, where $c_{0}=a_{1}=g_{0}^{*}+2 g_{1}^{*}+4 g_{2}^{*}$. We have [ ] $<2-q \leqslant 0, q \geqslant 2$, and thus $\hat{c}_{0}>0, q>2$, which implies $c_{0}>0, q>2$. Therefore $\mathrm{N}^{\prime}$ is satisfied for $q>2$. Condition $\mathrm{N} 2$ requires that $P(\xi, q)=$ $2 \gamma(q)(\xi-1)^{2} \geqslant 0,-1 \leqslant \xi \leqslant 1$, where

$$
\begin{aligned}
\gamma(q) & =q^{-5} \gamma_{1}(q) \gamma_{2}(q), \quad \gamma_{1}(q)=1 / 2\left[(2-q)-(2+q) e^{-q}\right], \\
\gamma_{2}(q) & =\left(-2+3 q-q^{2}\right)+(2-q) e^{-q} .
\end{aligned}
$$

As stated above, $\gamma_{1}(q)>0$ for $q>2$. But $\gamma_{2}(q)<-q^{2}+3 q-2=(q-2)(1-q)$ $\langle 0, q\rangle 2$; thus $\gamma(q)>0, q\rangle 2$, and $P(\xi, q) \geqslant 0$ for all $\xi,-1 \leqslant \xi \leqslant 1, q\rangle 2$, which completes the proof of $A$-stability.

The formula

$$
\begin{aligned}
& F_{3}^{*}:\left[\left(11-12 q^{-1}+6 q^{-2}\right)-e^{-q}\left(2-6 q^{-1}+6 q^{-2}\right)\right] y_{n+1} \\
& +\left[\left(-18+30 q^{-1}-18 q^{-2}\right)-e^{-q}\left(3+12 q^{-1}-18 q^{-2}\right)\right] y_{n} \\
& +\left[\left(9-24 q^{-1}+18 q^{-2}\right)-e^{-q}\left(-6-6 q^{-1}+18 q^{-2}\right)\right] y_{n-1} \\
& +\left[\left(-2+6 q^{-1}-6 q^{-2}\right)-e^{-q}\left(1-6 q^{-2}\right)\right] y_{n-2} \\
& -h\left\{\left[\left(6-11 q^{-1}+12 q^{-2}-6 q^{-3}\right)-e^{-q}\left(-2 q^{-1}+6 q^{-2}-6 q^{-3}\right)\right] y_{n+1}^{\prime}\right. \\
& +\left[\left(18 q^{-1}-30 q^{-2}+18 q^{-3}\right)-e^{-q}\left(6-3 q^{-1}-12 q^{-2}+18 q^{-3}\right)\right] y_{n}^{\prime} \\
& +\left[\left(-9 q^{-1}+24 q^{-2}-18 q^{-3}\right)-e^{-q}\left(6 q^{-1}+6 q^{-2}-18 q^{-3}\right)\right] y_{n-1}^{\prime} \\
& \left.+\left[\left(2 q^{-1}-6 q^{-2}+6 q^{-3}\right)-e^{-q}\left(-q^{-1}+6 q^{-3}\right)\right] y_{n-2}^{\prime}\right\}=0
\end{aligned}
$$

is $A_{\infty}$-stable [10] (i. e., $\mathrm{N}^{\prime}$ is satisfied) for $q \geqslant 5$. To prove it we show that $a_{0}$, $b_{0}, c_{0}=a_{1}-\left(a_{0} b_{1} / b_{0}\right)$, and $d_{0}=b_{1}$ are nonzero and of equal sign for $q \geqslant 5$.

First $a_{0}=6\left(1-e^{-q}\right)>0$ for any $q>0$. Then

$$
b_{0}=\left(18-12 q^{-1}\right)+\left(-6+12 q^{-1}\right) e^{-q} \geqslant\left(18-12 q^{-1}\right)+\left(-6+12 q^{-1}\right)=12>0
$$

${ }^{* *}$ By $g_{i}$ and $g_{i}^{*}$ we denote generic diagonal entries of $G_{i}$ and $G_{i}^{*}$, respectively. 
for $q \geqslant 2$. Next write $d_{0}=2 q^{-3}\left[p_{1}(q)+p_{2}(q) e^{-q}\right]$. One finds $p_{2}^{\prime}(q)>0$ for $q \geqslant 2, p_{2}(2)>0$, and thus $p_{2}(q)>0, q \geqslant 2$. Similarly, $p_{1}^{\prime}(q)>0, q \geqslant 4$; $p_{1}(5)>0$, and thus $p_{1}(q)>0$ and $d_{0}>0$ for all $q \geqslant 5$. Finally subject to condition $b_{0}>0$ which is satisfied for $q \geqslant 2$, we have

$$
c_{0}>0 \Leftrightarrow a_{1} b_{0}-a_{0} b_{1}=q^{2} N(q) / 48>0,
$$

where

$$
N(q)=\left(6 q^{2}-13 q+9\right)+(8 q-12) e^{-q}-(q-3) e^{-2 q} .
$$

For $q>3,8 q-12>0$ and $q-3>0$. Hence,

$$
N(q)>\left(6 q^{2}-13 q+9\right)-(q-3)=6 q^{2}-14 q+12>0
$$

for all $q \geqslant 5$, which completes the proof that $F_{3}^{*}$ is $A_{\infty}$-stable for $q \geqslant 5$.

For $q \geqslant 5, F_{3}^{*}$ possesses other properties of strong fixed- $h$ stability, as a numerical investigation of the relevant conditions showed. In particular it was found that $F_{3}^{*}$ is $A(\alpha)$-stable [8] for $\alpha \leqslant \alpha_{0}(q)$. The maximum angle $\alpha_{0}(q)$ increases from $\approx 84.4^{\circ}$ (1.4731 in radians) for $q=5$ to $\approx 86^{\circ}$ for $q=+\infty$, the latter representing the three-step BDF [9], [15]. Furthermore, $F_{3}^{*}$ is $A(a)$-stable [10] (i. e., fixed- $h$ stable for $\left.\operatorname{Re} q^{\prime}<-a\right)$ for any $a \geqslant a_{0}(q)$. We found that $a_{0}=.22435$ for $q=5$, that $a_{0}(q)$ is monotone decreasing and, for $q=+\infty, a_{0}=1 / 12=.08333$ [9]. Finally, for $q=5$ and $c=30.708, F_{3}^{*}$ is $A(r, c)$-stable [10] (i.e., fixed- $h$ stable in $\left.\left|q^{\prime}-c\right|>r\right)$ for any $r \geqslant r_{0}=31.14$. The center $c$ was chosen in such a way that, approximately, the circle $\left|q^{\prime}-c\right|=r$ is tangent to the image curve of $|w|=1$ by the map $q^{\prime}(w)=\rho(w) / \sigma(w)$ at its intersection point with the imaginary axis (so as to reduce to a "minimum" the area cut out of the left half $q$ '-plane by the circle). Again, as $q$ increases from $q=5$ the $A(r, c)$-stability constraint becomes weaker. For example, the BDF $(q=+\infty)$ is $A(r, c)$-stable with $c=6$ for any $r \geqslant r_{0}=6.31$ [10]. In Figure 1 , the image curve by $q^{\prime}(w)$ of $|w|=1$, is plotted for $q=5,6$, and $+\infty$ and the bounds of $A(\alpha)-A(a)$-, and $A(r, c)$-stability are shown for $q=5$.

For $q=+\infty$, the formulas $F_{k}^{*}$ are the BDF and are thus trivially $A_{\infty}$-stable for all $k$. Because of the continuous dependence of its coefficients on $q, F_{k}^{*}(q)$ for any given $k$ must possess this property for any sufficiently large value of $q$. Similarly, for $q=0$, the formulas $F_{k}$ and $F_{k}^{*}$ are Adams formulas which are stable [11] (in the limit $q^{\prime} \rightarrow 0$ ), and even fixed- $h$ stable for a bounded set of values of $q^{\prime} . F_{k}$ and $F_{k}^{*}$ must thus be stable for any sufficiently small, nonzero value of $q$.

Of course, $F_{k}^{*}(q)$, which for a large value of $q$ is $A_{\infty}$-stable, may not simultaneously be stable for that value of $q$. A well-known example of this are the BDF for $k \geqslant 7$ [9]. Similarly, the stable "near-Adams" formulas $F_{k}(q)$ and $F_{k}^{*}(q)$ associated with small values of $q$ are not $A_{\infty}$-stable. This lack of uniformity in the stability properties is, however, not a serious drawback for the following reason: In our 
matricial integration scheme, the stiff component solutions (corresponding to large values of $q=h d$ ) are treated by row-formulas fitted at large values of $q$. These

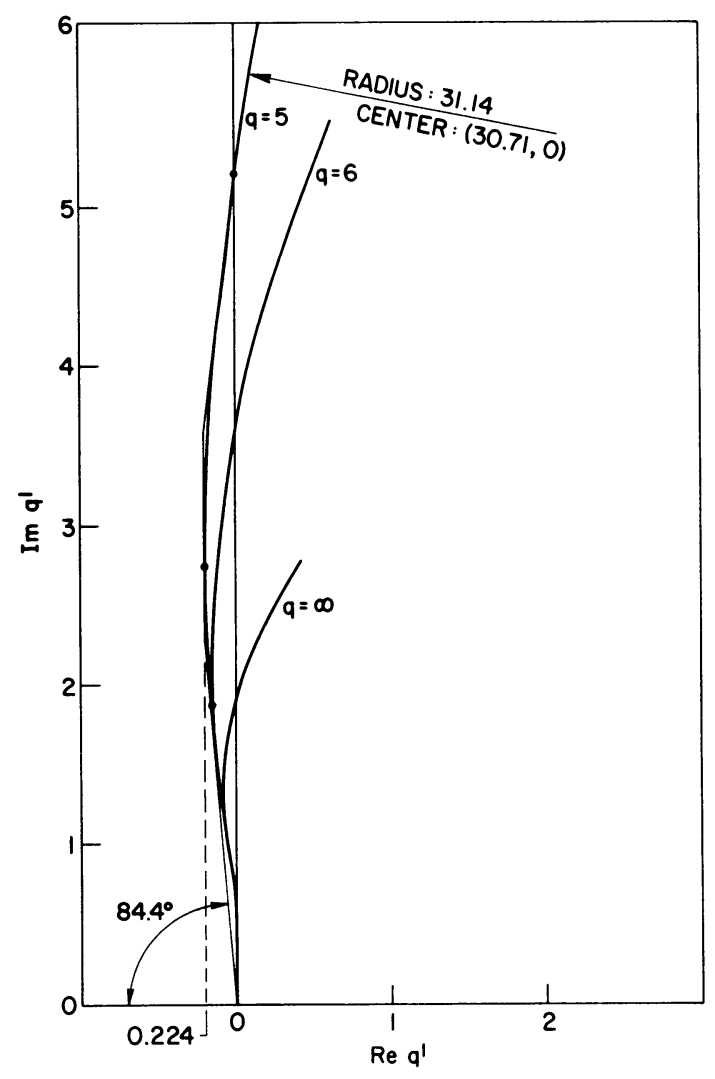

FIGURE 1. Stability domains for S3 as a function of $q$.

formulas then possess precisely the unconditional fixed- $h$ stability properties needed to control the stiff components. On the other hand, the slowly varying (smooth, nonstiff) components corresponding to small values of $q$ are treated by Adams-like formulas fitted at those small values of $q$. These latter formulas then possess precisely the stability-and limited fixed- $h$ stability-properties which are relevant for the smooth components.

As an exception to the lack of uniformity mentioned in the last paragraph, note that $F_{3}^{*}$ is stable for all $q, 0 \leqslant q \leqslant+\infty$. To show this let $\rho(w, q)$ be the cubic polynomial associated with the $\alpha$-coefficients of $F_{3}^{*}$ and let ${ }^{* * *} \hat{\rho}(w, q)=$ $\rho(w, q) /(w-1)$, a quadratic polynomial. Furthermore, let

$$
\hat{r}(z, q)=(z-1)^{2} \hat{\rho}((z+1) /(z-1))=2 c_{0}(q)+3 q c_{1}(q) z+3 q^{2} c_{2}(q) z^{2} .
$$

\footnotetext{
${ }^{* * *}$ By consistency, $\rho(w, q)$ possesses the linear factor $(w-1)$ for all $q$.
} 
With appropriate normalization, $c_{0}(q)=\left(6-9 q+5 q^{2}\right)+\left(-6+3 q+q^{2}\right) e^{-q}$, $c_{1}(q)=(-2+3 q)+(2-q) e^{-q}$, and $c_{2}(q)=1-e^{-q}$. Now apply the Routh criterion to $\hat{r}(z, q)$ : First $c_{2}(q)>0$ for $q>0$. Next, for $0<q \leqslant 3, c_{1}^{\prime}(q) \geqslant q>0$, $c_{1}(0)=0$, and thus $c_{1}(q)>0$ for $0<q \leqslant 3$. For $q \geqslant 3, c_{1}(q) \geqslant 2 q>0$; thus $c_{1}(q)>0$ for all $q, 0<q \leqslant+\infty$. Finally, for $q \geqslant q_{1}=(-3+\sqrt{33}) / 2$, we have $-6+3 q+q^{2} \geqslant 0$ and $c_{0}(q) \geqslant 6-9 q+5 q^{2}>0$. For $0<q \leqslant q_{1}$,

$$
c_{0}(q) \geqslant\left(6-9 q+5 q^{2}\right)+\left(-6+3 q+q^{2}\right)\left[1-q+\left(q^{2} / 2\right)\right]=\left(q^{3}+q^{4}\right) / 2>0
$$

which completes the proof.

\section{Algorithmic Implementation.}

A. Newton-Raphson Algorithm. Algorithms useful for solving stiff systems are generated by applying the Newton-Raphson (NR) method to the nonlinear difference equations associated with the formulas $\mathbf{F}_{k}^{*}[12]$. The derivation is along the lines of [24] and is given in detail in [16]. We give the result of this procedure for $k=3$. In this case, if a starting guess for the NR method is obtained by quadratic extrapolation from preceding solution values, the NR iteration may be stopped after one step [13].

Let

$$
\begin{array}{ll}
S_{0}^{*}=B_{3,0}^{*}, & S_{1}^{*}=-\left(B_{3,1}^{*}+B_{3,2}^{*}+B_{3,3}^{*}\right), \\
S_{2}^{*}=B_{3,2}^{*}+2 B_{3,3}^{*}, & S_{3}^{*}=-B_{3,3}^{*},
\end{array}
$$

where the $B_{3, i}^{*}, i=0, \cdots, 3$, are $(6 Q)^{-1}$ times the matrix equivalent of the coefficients $\beta_{3-i}$ of (3.3), respectively. For the autonomous case of (1.2), the one-step NR algorithm for $F_{3}^{*}$ is then defined by the following relations. Let

$$
\begin{gathered}
\Delta \tilde{y}=\nabla y+\nabla^{2} y, \quad \tilde{y}^{+}=y+\Delta \tilde{y}, \quad \tilde{\phi}^{+}=\phi\left(\tilde{y}^{+}\right), \\
\widetilde{\phi}_{y}^{+}=\phi_{y}\left(\tilde{y}^{+}\right), \quad \phi_{y}=\partial \phi / \partial y ;
\end{gathered}
$$

compute $\delta y^{+}$by solving

$$
\left(I-h S_{0}^{*} \widetilde{\phi}_{y}^{+}\right) \delta y^{+}=-\Delta \tilde{y}-\left(I-e^{-Q}\right) y+h\left(S_{0}^{*} \tilde{\phi}^{+}-S_{1}^{*} \phi-S_{2}^{*} \nabla \phi-S_{3}^{*} \nabla^{2} \phi\right)
$$

correct by

$$
y^{+}=\tilde{y}^{+}+\delta y^{+}, \quad \nabla^{2} y^{+}=\nabla^{2} y+\delta y^{+}, \quad \nabla y^{+}=\nabla y+\nabla^{2} y^{+}
$$

and reevaluate:

$$
\phi^{+}=\phi\left(y^{+}\right), \quad \nabla \phi^{+}=\phi^{+}-\phi, \quad \nabla^{2} \phi^{+}=\nabla \phi^{+}-\nabla \phi .
$$

As a starting procedure in conjunction with this algorithm, one may use implicit RungeKutta methods (see e.g., [25]).

B. Analysis of the Local Truncation Error. The error analysis given hereafter is similar to that of [17]. However, we recall that our aim is to derive formulas which are accurate for sufficiently small values of $h \phi$ or of a derivative thereof but for arbitrarily large values of $Q=h D$. Therefore, in analyzing the local truncation error, it is unreasonable to make a complete expansion of this error in powers of $h$ (including 
the coefficients of the formula via their dependence on $Q$ ). Instead we shall adopt the point of view that $Q$ is an arbitrarily large parameter which we keep constant independently of $h$. We can, of course, think of this situation as describing a sequence of problems in which we simultaneously vary $h$ and $D$ in an appropriate way.

The formulas we derive hereafter are applicable to the smooth solutions encountered during the asymptotic phase but do not apply to the rapid transients. ${ }^{\dagger}$ In fact, for constant $q>0$ and $h \rightarrow 0$, we have $d \rightarrow+\infty$ and in this limit the transient solutions are not smooth. This is exemplified by the case where $\phi(x, y) \equiv 0$, $y^{\prime}=-d y$, and the solution $y(x)=y_{0} e^{-d x}$ for $d=+\infty$ is a step function.

Consider the linear differential difference operator (with $k=p+1$ for given $p$ )

$$
\mathbf{L}_{p+1}=\sum_{j=-p}^{1} \alpha_{j+p}(Q) E^{j}-h \sum_{j=-p}^{0} \beta_{j+p}(Q) E^{j} \frac{d}{d x}
$$

associated with $\mathbf{F}_{p+1}$, where $E$ is the shift operator. The order of $\mathbf{L}_{p+2}$ exceeds that of $\mathbf{L}_{p+1}$ by one. Therefore, the principal part of the local truncation error of $\mathbf{L}_{p+1}$ is the same as that of $\mathbf{L}_{p+1}-\mathbf{L}_{p+2}$. We find that

$$
\left(\mathbf{L}_{p+1}-\mathbf{L}_{p+2}\right) y(x)=h G_{p+1} \nabla^{p+1}\left[y^{\prime}(x)+D y(x)\right],
$$

where we have substituted $y^{\prime}+D y$ for $\phi$. For a smooth solution $y(x)$ of (1.2), this yields

$$
\begin{aligned}
\left(\mathbf{L}_{p+1}-\mathbf{L}_{p+2}\right) y(x) & =Q G_{p+1} \nabla^{p+1} y(x)+h G_{p+1} \nabla^{p+1} y^{\prime}(x) \\
& =h^{p+1} Q G_{p+1} y^{(p+1)}(x)+O\left(h^{p+2}\right),
\end{aligned}
$$

and, from what we have said, it follows that

$$
\mathbf{L}_{p+1} y(x)=h^{p+1} Q G_{p+1} y^{(p+1)}(x)+O\left(h^{p+2}\right) .
$$

Therefore, $\mathbf{F}_{p+1}$ has order of accuracy $p$ when $Q \neq 0$, and $p+1$ when $Q=0$; as mentioned before, the latter represents the well-known result for open Adams formulas. In the general case, the coefficient of the principal error term of $\mathbf{L}_{p+1}$ is $C_{p+1}=$ $Q G_{p+1}$ according to (4.1).

The error analysis for $\mathbf{F}_{p}^{*}$ is analogous to that of $\mathbf{F}_{p+1}$. If (with $k=p$ for given $p$ )

$$
\mathbf{L}_{p}^{*}=\sum_{j=-(p-1)}^{1} \alpha_{j+p-1}^{*}(Q) E^{j}-h \sum_{j=-(p-1)}^{1} \beta_{j+p-1}^{*}(Q) E^{j} \frac{d}{d x}
$$

is the operator associated with $\mathbf{F}_{p}^{*}$, we find that

$$
\mathbf{L}_{p}^{*} y(x)=h^{p+1} Q G_{p+1}^{*} y^{(p+1)}(x)+O\left(h^{p+2}\right) .
$$

Thus, for $Q \neq 0, \mathrm{~F}_{p}^{*}$ has order of accuracy $p$. For $Q=0$ we recover the wellknown result that the $p$-step closed Adams formula, with which $\mathbf{F}_{p}^{*}$ is identical in

$\dagger$ This remark relates to the footnote of Section 1. 
this case, is of order $p+1$. The coefficient of the principal error term of $\mathbf{L}_{p}^{*}$ is $C_{p+1}^{*}=Q G_{p+1}^{*}$.

We can now write down approximate expressions for the local truncation errors of $\mathbf{F}_{p+1}$ and $\mathbf{F}_{p}^{*}$ by the method of Milne [26]. We define a predicted value $\bar{y}_{n+1}^{0}$ by letting $\mathrm{L}_{p+1} \bar{y}_{n}^{0}=0$ where, at $x_{n-p}, x_{n-p+1}, \cdots, x_{n}, \mathrm{~L}_{p+1}$ operates on the exact solution $y(x)$. One finds that

$$
\alpha_{p+1}\left[\bar{y}_{n+1}^{0}-y\left(x_{n+1}\right)\right] \simeq-h^{p+1} C_{p+1} y^{(p+1)}\left(x_{n}\right)
$$

where $\simeq$ denotes equality up to and including $O\left(h^{p+1}\right)$. Similarly, we define a corrected value $y_{n+1}^{1}$ by $\mathbf{L}_{p}^{*} y_{n}^{1}=0$, where for $y_{n+1}^{\prime}$ we substitute $\bar{\phi}_{n+1}^{0}-D \bar{y}_{n+1}^{0}$, with $\bar{\phi}_{n+1}^{0}=\phi\left(x_{n+1}, \bar{y}_{n+1}^{0}\right)$. Then

$$
\alpha_{p}^{*}\left[y_{n+1}^{1}-y\left(x_{n+1}\right)\right] \simeq-h^{p+1} C_{p+1}^{*} y^{(p+1)}\left(x_{n}\right) .
$$

We can eliminate $y\left(x_{n+1}\right)$ between (4.2) and (4.3) and solve for $y^{(p+1)}\left(x_{n}\right)$ :

(4.4) $y^{(p+1)}\left(x_{n}\right)=h^{-(p+1)}\left[\alpha_{p+1}^{-1} C_{p+1}-\alpha_{p}^{*-1} C_{p+1}^{*}\right]^{-1}\left(y_{n+1}^{1}-\bar{y}_{n+1}^{0}\right)+O(h)$.

Obviously, $\alpha_{p+1}$ is the identity matrix, whereas

$$
\alpha_{p}^{*}=I-Q B_{p, 0}^{*} \text {. }
$$

From (4.2) and (4.4), we deduce an estimate for the local truncation error of $\mathbf{F}_{p+1}$,

$$
\bar{y}_{n+1}^{0}-y\left(x_{n+1}\right) \approx \alpha_{p}^{*} \hat{G}_{p+1}\left[\hat{G}_{p+1}^{*}-\alpha_{p}^{*} \hat{G}_{p+1}\right]^{-1}\left(y_{n+1}^{1}-\bar{y}_{n+1}^{0}\right)
$$

where we have substituted $\hat{G}_{p+1}=(I+Q) G_{p+1}$ for $G_{p+1}$ and $\hat{G}_{p+1}^{*}=$ $(I+Q) G_{p+1}^{*}$ for $G_{p+1}^{*}$. An expression equivalent to (4.5) would be obtained if we were to replace $\hat{G}$ by $G$ and $\hat{G}^{*}$ by $G^{*}$. But, as $q \rightarrow+\infty$, the quantities $\hat{G}(q) \sim$ $q G(q)$ and $\hat{G}^{*}(q) \sim q G^{*}(q)$ tend to finite, nonzero limits as we have seen in Section 2. On the other hand $\hat{G} \sim G$ and $\hat{G}^{*} \sim G^{*}$ for $q \rightarrow 0$, and, in this limit, these quantities in turn have reasonable limits. Thus, relation (4.5) is in a convenient form for computational purposes.

In a very similar manner as for the formula $\mathbf{F}_{p+1}$ we obtain an estimate for the local truncation error of $\mathbf{F}_{p}^{*}$ :

$$
y_{n+1}^{1}-y\left(x_{n+1}\right) \approx \hat{G}_{p+1}^{*}\left[\hat{G}_{p+1}^{*}-\alpha_{p}^{*} \hat{G}_{p+1}\right]^{-1}\left(y_{n+1}^{1}-\bar{y}_{n+1}^{0}\right) .
$$

The error estimates (4.5) and (4.6) can be used for step size control or, as in the next subsection, for defining modifier formulas associated with the predictor and corrector formulas considered above.

C. Predictor-Corrector Algorithms. The relations (4.5) and (4.6) can be used to define modified predicted and corrected values. This is done by letting these modified values play the role of $y\left(x_{n+1}\right)$ in (4.5) and (4.6), by replacing the $\approx$ sign of these relations by the strict equality sign and, in the modifier of the predictor, by shifting the abscissa of $\left(\bar{y}^{0}-y^{1}\right)$ back by $h$. In this sense we arrive at the following one-step predictor-modifier-corrector-modifier (PMCM) algorithm: 


$$
\begin{aligned}
& \bar{y}_{n+1}^{0}=e^{-Q_{y_{n}}+h} \sum_{i=-(p-1)}^{1} B_{p, i+p-1} \phi_{n-(p-1)-i}, \\
& \bar{y}_{n+1}=\bar{y}_{n+1}^{0}+\alpha_{p}^{*} \hat{G}_{p+1}\left[\hat{G}_{p+1}^{*}-\alpha_{p}^{*} \hat{G}_{p+1}\right]^{-1}\left(\bar{y}_{n+1}^{0}-y_{n+1}^{1}\right) \text {, } \\
& y_{n+1}^{1}=e^{-Q} y_{n}+h\left[B_{p, 0}^{*} \bar{\phi}_{n+1}+\sum_{i=-(p-2)}^{1} B_{p, p-1+i}^{*} \phi_{n+1-i}\right] \text {, } \\
& y_{n+1}=y_{n+1}^{1}+\hat{G}_{p+1}^{*}\left[\hat{G}_{p+1}^{*}-\alpha_{p}^{*} \hat{G}_{p+1}\right]^{-1}\left(\bar{y}_{n+1}^{0}-y_{n+1}^{1}\right) \text {, }
\end{aligned}
$$

where $\bar{\phi}=\phi(x, \bar{y}), \hat{G}_{p+1}=(I+Q) G_{p+1}$, and $\hat{G}_{p+1}^{*}=(I+Q) G_{p+1}^{*}$. In the limit $Q \rightarrow 0$, the component relations of (4.7) through (4.10) become identical with Adams PMCM algorithms (see e.g., [27] for the cases $p=2,3$ ).

In lieu of the PMCM procedure, it may be advantageous in some cases to use an algorithm in which the formulas (4.7) and (4.8) are combined with a number of applications of the corrector formula,

$$
\begin{array}{r}
y_{n+1}^{i}=e^{-Q_{y_{n}}+h\left[B_{p, 0}^{*} \phi_{n+1}^{i-1}+\sum_{j=-(p-2)}^{1} B_{p, p-1-j}^{*} \phi_{n+1-j}\right]}, \\
i=1,2, \cdots,
\end{array}
$$

where $\phi^{l}=\phi\left(x, y^{i}\right)$ and $\phi^{0}=\phi$. The iteration may be stopped when an appropriate measure for the difference between two consecutive corrected values of $y$ becomes smaller than a prescribed threshold. We refer to this as the predictor-modifieriterated-corrector (PMIC) procedure.

D. Remark. If one tries to compute the quantities $G_{i}$ and $G_{i}^{*}$ by the closed expressions of Section 2 the results, because of numerical cancellation, are strongly affected by rounding errors in the limit where a component $q$ of $Q$ satisfies $|q| \ll 1$. It is preferable in this case to compute such components of $G_{i}$ or $G_{i}^{*}$ by power series expansions [16]. It was found by numerical experimentation on an APL system using 56-bit hexadecimal arithmetic, that in computing the quantities $g_{i}^{*}, 0 \leqslant i \leqslant 3$, a natural separation value $q_{s}$, between the range $0 \leqslant q \leqslant q_{s}$ in which the series evaluation should be used and the range $q_{s} \leqslant q$ in which the closed expressions seem more accurate, is $q_{s}=0.0085$.

5. Numerical Results. The Newton-Raphson algorithm associated with $\mathbf{F}_{3}^{*}$-referred to as S3 in the following-was applied' to two stiff test problems. The first of these is (a slightly rescaled version of) the first example listed in the survey paper of Bjurel et al. [28, Section 4, p. 1], and is due to Fowler and Warten [29]. This problem, which will be referred to as $P 1$, is defined by the equations and initial conditions

$$
\text { P1 } \begin{cases}y_{1}^{\prime}=-2000 y_{1}+1000 y_{2}+1000, & y_{1}(0)=0, \\ y_{2}^{\prime}=y_{1}-y_{2}, & y_{2}(0)=0 .\end{cases}
$$

Its exact solution is given in [16]. Near $x=0$ the solution to P1 has a "boundary 
layer" in the $y_{1}$-component, of amplitude $\approx 0.5$. To avoid this area, the numerical integration was actually carried out in the interval $1 \leqslant x \leqslant 4$, using the exact solution values at $x_{0}=1, x_{1}=1+h$, and $x_{2}=1+2 h$ as starting values. The relative accumulated truncation error in absolute value was computed and maximized over the abscissa values $x_{i}=1+i \times 0.6, i=0, \cdots, 5$. The second component (the larger one) of this error is plotted in Figure 2 as a function of $h$. The curve labelled S3 represents the results by the third order algorithm S3 using $d_{1}=2000$ and $d_{2}=1$. For comparison, the results of the third order algorithm A3 of [24] is plotted under this label, and the results obtained by the BDF with $k=p=3$, implemented as S3 with $d_{1}=d_{2}=10^{5}$ (in place of $+\infty$ ), is plotted as curve BDF3. We find that, for this linear problem with constant coefficients, the algorithm S3 is considerably more accurate than either BDF3 or $\mathrm{A} 3$, despite the fact that the off-diagonal terms are not small compared to the diagonal ones. It should be remembered, however, that $\mathrm{A} 3$ was designed to be $A$-stable whereas S3 is not $A$-stable. The order of accuracy reflects itself in the slope of the approximately straight-line curves. The theory predicts that the accumulated truncation error is $O\left(h^{p}\right), p=3$. In accordance with this the three curves. have a slope of $-p \approx-3$ in this manner of plotting.

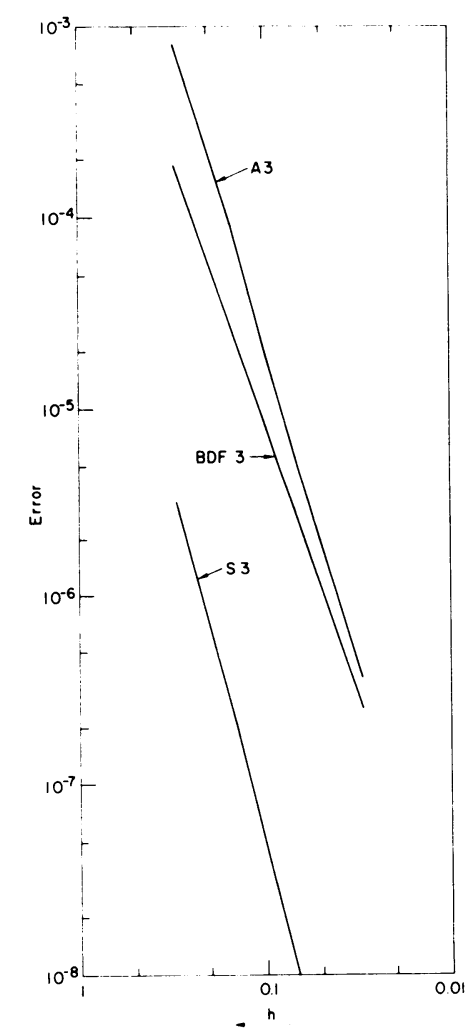

FIGURE 2. Accumulated truncation errors for test problem P1. 
As a second test problem we solved the nonlinear system [24]

$$
\text { P2 }\left\{\begin{array}{l}
y_{1}^{\prime}=0.01-\left[1+\left(1000+y_{1}\right)\left(1+y_{1}\right)\right]\left(0.01+y_{1}+y_{2}\right), \\
y_{2}^{\prime}=0.01-\left(1+y^{2}\right)\left(0.01+y_{1}+y_{2}\right),
\end{array}\right.
$$

from $x=1$ through $x=81$. As starting values near $x=1$ we used values of the "exact" solution defined by $y_{1}(0)=y_{2}(0)=0$ and computed by the well-known fourth-order Runge-Kutta method with an extremely small step, $h=.002$. The eigenvalues are $\lambda_{1} \approx-981, \lambda_{2} \approx-2 \times 10^{-5}$ at $x=1$, and $\lambda_{1} \approx-185, \lambda_{2} \approx-10^{-3}$ at $x=81$. The numerical results of applying the algorithms S3 with $d_{1}=1011.01$ and $d_{2}=1, \mathrm{~A} 3$, and $\mathrm{BDF} 3$ to $\mathrm{P} 2$ are plotted in Figure 3 in a similar manner as for $\mathrm{P} 1$ in Figure 2. Again the algorithm S3 proves to be more accurate than A3 and BDF3, although the difference in this nonlinear problem is not as great as in P1. For both problems, calculations have been carried out with values of $D$ other than the two associated with the curves labelled A3 and BDF, respectively. The

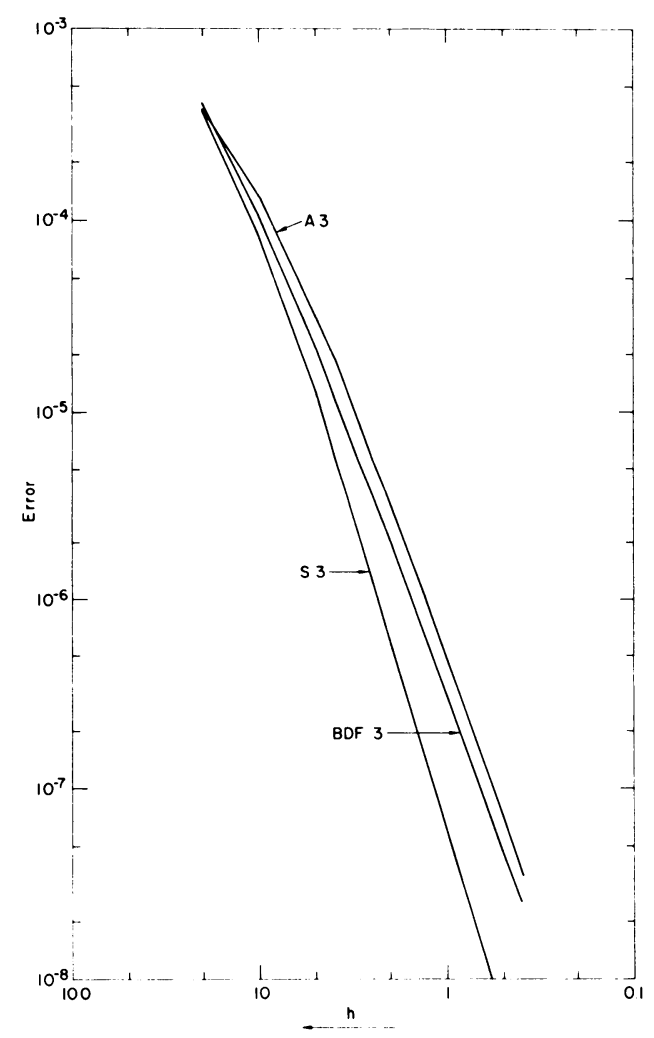

FIGURE 3. Accumulated truncation errors for test problem P2.

results generally showed a low sensitivity to reasonable changes of $D$ within the stability constraints given in Section 3 . 
The third-order PMCM and PMIC procedures were tested on five stiff or diagonally dominant, nonstiff problems [16]. In summary, the following observations were made in these tests: As far as stiff problems are concerned, the PMIC procedure yielded useful, though somewhat erratic results even with relatively large integration steps. Instead, as expected, open methods such as PMCM, Adams, and explicit Runge-Kutta procedures broke down for all but the smallest step sizes used. With small integration steps, PMCM appeared to be more accurate than the corresponding third-order Adams procedure (which is the special case of PMCM fitted at $Q=0$ ), or the standard fourth-order Runge-Kutta method. Similarly, for nonstiff problems and for intermediate step sizes at which Adams and Runge-Kutta are still stable, the exponentially fitted algorithms PMCM and PMIC proved to be more accurate than the former methods. As one might expect, the gain was greater in linear than in nonlinear problems.

Acknowledgment. The authors would like to express their gratitude to the referee for his very valuable suggestions.

IBM Corporation

System Development Division

Kingston, New York 12401

IBM Thomas J. Watson Research Center

Yorktown Heights, New York 10598

1. E. F. SARKANY \& W. E. BALL, "A predictor-corrector modification of the Cohen-FlattCertaine method for solving differential equations," Proceedings Joint Conference on Mathematical and Computer Aids to Design, Anaheim, Calif., 1969, p. 336.

2. J. CERTAINE, "The solution of ordinary differential equations with large time constants," Mathematical Methods for Digital Computers, A. Ralston and H. S. Wilf (Editors), vol. 1, Wiley, New York, 1960, pp. 128-132. MR 22 \#8691.

3. E. R. COHEN \& H. P. FLATT, "Numerical solution of quasilinear equations," Codes for Reactor Computations, International Atomic Energy Agency, Vienna, 1961, pp. 461-484.

4. K. G. GUDERLEY \& C. C. HSU, "A predictor-corrector method for a certain class of stiff differential equations," Math. Comp., v. 26, 1972, pp. 51-69. MR 45 \#8001.

5. W. LINIGER, "Global accuracy and $A$-stability of one- and two-step integration formulae for stiff ordinary differential equations," Conference on the Numerical Solution of Differential Equations (Dundee, 1969), Lecture Notes in Math., vol. 109, Springer-Verlag, Berlin, 1969, pp. $188-$ 193.

6. C. F. CURTISS \& J. O. HIRSCHFELDER, "Integration of stiff equations," Proc. Nat. Acad. Sci. U.S. A., v. 38, 1952, pp. 235-243. MR 13, 873.

7. G. G. DAHLQUIST, “A special stability criterion for linear multistep methods," Nordisk Tidskr. Informationsbehandling (BIT), v. 3, 1963, pp. 27-43. MR 30 \#715.

8. O. B. WIDLUND, "A note on unconditionally stable linear multistep methods," Nordisk Tidskr. Informationsbehandling (BIT), v. 7, 1967, pp. 65-70. MR 35 \#6373.

9. C. W. GEAR, "The automatic integration of stiff ordinary differential equations (With discussion)," Information Processing 68 (Proc. IFIP Congress, Edinburgh, 1968), vol. I: Mathematics, Software, North-Holland, Amsterdam, 1969, pp. 187-193. MR 41 \#4808. 
10. F. ODEH \& W. LINIGER, "A note on unconditional fixed- $h$ stability of linear multistep methods," Computing (Arch. Elektron. Rechnen), v. 7, 1971, pp. 240-253. MR 45 \#8006.

11. G. G. DAHLQUIST, "Stability and error bounds in the numerical integration of ordinary differential equations," Kungl. Tekn. Högsk. Stockholm, No. 130, 1959, 87 pp. MR 21 \#1706.

12. W. LINIGER \& R. A. WILLOUGHBY, "Efficient integration methods for stiff systems of ordinary differential equations," SIAM J. Numer. Anal., v. 7, 1970, pp. 47-66. MR 41 \#4809.

13. W. LINIGER, "A stopping criterion for the Newton-Raphson method in implicit multistep integration algorithms for nonlinear ordinary differential equations," Comm. $A C M$, v. 14, 1971, pp. 600-601. MR 44 \#7754.

14. R. K. JAIN, "Some $A$-stable methods for stiff ordinary differential equations," Math. Comp., v. 26, 1972, pp. 71-77. MR 46 \#2869.

15. S. NQRSETT, "An $A$-stable modification of the Adams-Bashforth methods," Conference on Numerical Solution of Differential Equations (Dundee, 1969), Lecture Notes in Math., vol. 109, Springer-Verlag, Berlin, 1969, pp. 214-219. MR 42 \#2673.

16. E. F. SARKANY \& W. LINIGER; Exponential Fitting of Matricial Multistep Methods for Ordinary Differential Equations, IBM Report RC 4149, Dec. 8, 1972.

17. P. HENRICI, Discrete Variable Methods in Ordinary Differential Equations, Wiley, New York, 1962, Chap. 5. MR 24 \#B1772.

18. L. G. KELLY, Handbook of Numerical Methods and Applications, Addison-Wesley, Reading, Mass., 1967, p. 39.

19. G. PETIT BOIS, Tables of Indefinite Integrals, Dover, New York, 1961, p. 145. MR 23 \#A256.

20. Op. cit., Ref. 17, p. 222.

21. G. G. DAHLQUIST, "Convergence and stability in the numerical integration of ordinary differential equations," Math. Scand., v. 4, 1956, pp. 33-53. MR 18, 338.

22. W. LINIGER, "A criterion for $A$-stability of linear multistep integration formulae," Computing (Arch. Elektron. Rechnen), v. 3, 1968, pp. 280-285. MR 39 \#1120.

23. F. R. GANTMAHER, The Theory of Matrices, GITTL, Moscow, 1953; English transl., Chelsea, New York, 1959, Vol. 2, Chap. 15. MR 16, 438; 21 \#6372c.

24. W. LINIGER \& F. ODEH, “ $A$-stable accurate averaging of multistep methods for stiff differential equations," IBM J. Res. Develop., v. 16, 1972, pp. 335-348.

25. R. H. ALLEN \& C. POTTLE, "Stable integration methods for electronic circuit analysis with widely separated time constants," Proceedings Sixth Allerton Conference on Circuit Design and System Theory, University of Illinois, 1967, pp. 534-543.

26. Op. cit., Ref. 17, p. 255.

27. L. LAPIDUS \& J. H. SEINFELD, Numerical Solution of Ordinary Differential Equations, Math. in Sci. and Engineering, vol. 74, Academic Press, New York, 1971, p. $182 . \quad$ MR 43 \#7073.

28. G. BJUREL, et al., Survey of Stiff Ordinary Differential Equations, Report NA 70.11, Roy. Inst. of Tech., Stockholm, Sweden.

29. M. E. FOWLER \& R. M. WARTEN, "A numerical integration technique for ordinary differential equations with widely separated eigenvalues," IBM J. Res. Develop., v. 11, 1967, pp. 537-543. MR 35 \#7586. 